\title{
Regulating Risk or Risking Regulation? Construal Levels and Depletion Effects in the Processing of Health Messages
}

\author{
NIDHI AGRAWAL \\ ECHO WEN WAN*
}

\begin{abstract}
The depletion effect occurs when individuals who exert self-control in a previous task (i.e., depleted individuals) exhibit less self-control on a subsequent task relative to individuals who did not previously exert self-control. This article presents two experiments that implicate construal levels to understand the processes underlying depletion effects in the context of consumer health. At low-level construals, individuals rely on resource accessibility cues (e.g., feelings of tiredness) to determine self-control. Hence, they exert less self-control only when they assess themselves as depleted, manifesting the depletion effect. High-level construals reduce the resource focus and enhance a goal focus, which diminishes and even reverses the depletion effect.
\end{abstract}

$\mathrm{C}$ onsumers often need to exert self-control in multiple activities in succession. For example, after persisting at a vigorous evening workout, a consumer might face the challenge of resisting candy in favor of carrots for a predinner snack. Past research has examined how a previous act of self-control affects subsequent self-control. For example, studies have shown that suppressing one's thoughts undermines one's subsequent control on impulse purchases (Vohs and Faber 2007). Likewise, resisting an impulse leads to excessive alcohol consumption (Muraven, Collins, and Nienhaus 2002). The phenomenon in which individuals reduce control on a self-control task after having exerted great self-control on an immediately preceding task is referred to as the depletion effect (Muraven, Tice, and Baumeister 1998).

Substantial research has demonstrated the depletion effect in diverse domains (e.g., impulse purchase [Vohs and Faber 2007], food consumption [Baumeister et al. 1998]). Typically in these studies, participants perform two tasks consecutively: the initial task requires either substantial (de-

*Nidhi Agrawal (nidhi-agrawal@kellogg.northwestern.edu) is assistant professor at the Kellogg School of Management, Northwestern University, 2001 Sheridan Road, Evanston, IL 60208-2001. Echo Wen Wan (ewan@ business.hku.hk) is assistant professor in marketing at the University of Hong Kong. Order of authorship is alphabetical and reflects equal contributions by both authors. The authors would like to thank Brian Sternthal and Angela Lee for their comments on earlier drafts of this manuscript.

John Deighton served as editor and Jennifer Aaker served as associate editor for this article.

Electronically published February 27, 2009 pleting) or little (non-depleting) self-control, and the second task tests the focal self-control. For example, Vohs and Faber (2007) asked participants to complete a depleting or nondepleting initial task (e.g., suppressing thoughts or not having to suppress thoughts) and then provided them the opportunity to make an unplanned (impulse) purchase in the second task. Findings were consistent with the depletion effect: depleted participants exhibited poorer self-control in impulse purchase situations than did non-depleted participants.

Resource depletion theory proposes that depletion effects occur because of the limited availability of self-control resources-a pool of psychological resources that individuals use to overcome default impulsive responses and handle trade-offs in self-control activities (Muraven and Baumeister 2000). Expending resources on one arduous self-control activity reduces the resources available for a subsequent selfcontrol task and thus undermines the performance on this task. Supporting this explanation, previous research has shown that depletion effects can be overcome by replenishing resources temporarily (Gailliot et al. 2007) or expanding the resource pool over time (Muraven, Baumeister, and Tice 1999).

We propose an alternative process by which depletion effects might occur. We suggest that depletion effects might be due to the way self-control resources are allocated. We propose that exerting self-control leads individuals to focus on the limited resources they may be able to devote to a subsequent self-control task and thus reduces the further allocation. If this focus on lack of resources shifted to goals, even depleted individuals would subsequently exert ade- 
quate self-control. Hence, the depletion effect occurs because individuals focus on the current lack of resources and not on goals. We employ construal level theory to test our proposed process. Levels of construal systematically influence individuals' tendency to focus on resources or goals (Trope and Liberman 2003). Specifically, we propose that lower construal levels make the role of resource accessibility in self-control more salient and lead to depletion effects. In contrast, higher construal levels emphasize goals while diluting the focus on the accessibility of resources in determining behavior and consequently diminish depletion effects.

Two experiments examined the processes underlying the interaction between depletion effects and construal levels in a health communication context. Health messages often attract consumers' attention by highlighting their high risk of contracting a disease. Building on the health risk literature, we suggest that processing health messages that convey high (vs. low) levels of risk consumes more self-control resources and thus will undermine subsequent self-control in performing health behaviors unrelated to the depleting message. If exposure to high-risk messages undermines subsequent selfcontrol, it is important to identify factors that will mitigate the negative repercussions of high-risk health messages. Testing our predictions in the health domain allows us to identify interventions that may curtail the harmful effects of high-risk health messages.

\section{CONSTRUAL LEVELS AND DEPLETION EFFECTS}

Although the current theories that delve into the depletion effect suggest that it occurs because of limited resources, there is emerging evidence that depletion effects may not necessarily occur due to a dearth of resources. For example, it has been found that providing monetary incentives $(\mathrm{Mu}-$ raven and Slessareva 2003) or accurate performance feedback (e.g., providing a clock on a timed self-control task; Wan and Sternthal 2008) can enhance self-control for depleted individuals and thus eliminate the depletion effect. Building on these recent findings, we propose that depletion effects occur because of a tendency to focus on the lack of resources while exerting self-control in consecutive tasks. If this focus shifted from resources to goals, depleted participants would engage in greater self-control. It is possible that by providing participants with accurate feedback or monetary incentives, previous studies might have enhanced goal salience. To directly test the proposition that focusing on goals might diminish the depletion effect, we employ construal levels, a factor that could systematically influence individuals' focus on resources versus goals (Liberman and Trope 1998).

Construal level theory (CLT; Trope and Liberman 2003) posits that the same event or object can be represented at multiple levels. Higher-level construals highlight central goals associated with an event. In contrast, lower-level construals highlight means and resources (Liberman and Trope
1998; Sagristano, Trope, and Liberman 2002; Trope, Liberman, and Wakslak 2007). For example, Liberman and Trope (1998) found that construing an event (e.g., going to a concert) at higher levels emphasized goals (e.g., enjoying music played by a band at the concert) rather than means (e.g., price of the concert ticket). Conversely, construing the same event at lower levels emphasized means rather than goals. Following past research on action identification and construal levels (Vallacher and Wegner 1987), Liberman and Trope (1998) conducted a series of studies showing that temporally distant events and events construed at higher levels focus more on goals or "why" aspects, whereas proximal events and events construed at lower levels focus on means or "how" aspects of the event.

Recently, Fujita et al. (2006) examined how construal levels might influence self-control. They proposed that lower levels of construal highlight local goals (e.g., playing a video game and having fun now; experiment 4) and hinder selfcontrol, whereas higher levels of construal highlight global goals (e.g., doing better academically by studying) and facilitate self-control. Their conceptualization argues that construal levels enhance or undermine self-control by highlighting different goals. However, it is not clear whether a goal-based analysis would apply in consecutive self-control contexts such as depletion effects (Vohs and Schmeichel 2003). Acknowledging that their local versus global goals conceptualization "is largely mute with respect to ego-depletion models of self-control," Fujita et al. (2006, 364-65) called for future research "both theoretically and empirically, on integrating the two theoretical approaches [of depletion effects and CLT]." In response to this call for research, we draw upon the goals-means distinction in CLT to make specific predictions about how construal levels will affect selfcontrol in situations involving resource depletion. We propose that construal levels will influence self-control in consecutive tasks through their differential emphasis on means versus goals.

\section{Depletion Effects under Lower Levels of Construal}

Lower-level construals foster greater attention to effort and current access to resources. For depleted individuals, a focus on resources increases the likelihood of dwelling on current feelings of fatigue and the current resource limitation, resulting in a desire to quit. For non-depleted individuals, a focus on means and resources leads to an assessment of plentiful resources, which does not discourage subsequent self-control. Therefore, we expect to observe depletion effects at lower construal levels.

H1a: At lower construal levels, depleted individuals will show poorer subsequent self-control than non-depleted individuals (depletion effect).

Moreover, we theorize that lower-level construals lead individuals to focus on resources and feelings of fatigue. For depleted participants, thinking about tiredness highlights 
their constrained ability to perform the subsequent task, which results in impaired self-control. Hence, at lower construal levels, a focus on tiredness should mediate the effect of initial resource depletion on subsequent self-control. This effect should not occur at higher levels of construal because of a reduced focus on means and resources.

H1b: At lower construal levels, the focus on resources (e.g., tiredness) will mediate the effect of initial depletion on subsequent self-control.

\section{Depletion Effects under Higher Levels of Construal}

Higher levels of construal foster a focus on goals and dilute the focus on resources. At higher levels of construal, individuals' self-control is determined by the extent to which a task might help in achieving a higher-level goal (Fujita et al. 2006). Hence, we predict that at higher levels of construal, regardless of initial depletion, individuals will exert self-control on the second task, depending on its relevance to their goals. When a task is important to the pursuit of higher-level goals, participants at higher-level construals would value the higher-level benefits of this task and exert adequate self-control. When a task is unimportant to the pursuit of higher-level goals, participants at higher-level construals should show decreased self-control. Lower levels of construal are not sensitive to higher-level goals. Hence, the importance of the second task should not affect participants' self-control.

H2a: At higher construal levels, individuals will exert greater self-control on a subsequent task of high rather than low importance, regardless of initial depletion.

H2b: At higher construal levels, perceived higherlevel benefits from performing the second selfcontrol task will mediate subsequent self-control.

Hypotheses $2 \mathrm{a}$ and $2 \mathrm{~b}$ present scenarios in which the higher-level benefit of the second task is clear. However, it is likely that the importance of the second task may be unclear or ambiguous. When task importance or related cues that inform individuals regarding their effort goals are absent, individuals may rely on salient or accessible recent experiences to infer such goals (Mussweiler 2003). In the context of depletion effects, participants might rely on their most salient and recent self-control experience (i.e., the initial task) to infer their goals regarding the amount of effort to be expended on the second task (Kruger et al. 2004; Wan and Sternthal 2008). In other words, at higher levels of construal, when the importance of the second task is unclear, participants who completed a depleting (non-depleting) task might use the greater (lower) effort demanded by the initial task as a cue to set their goals for the second task. Hence, those in the depleting condition would work toward achiev- ing a higher bar on the second task, relative to those in the non-depleting condition. This would result in substantial self-control when the initial task is depleting and little selfcontrol when the initial task is non-depleting. Hence, in the absence of a clear indicator of the importance of the second task, the reliance on the initial task to infer subsequent resource allocation would result in the reversal of the depletion effect.

H2c: At higher construal levels, when the importance of performing the second self-control task is unclear, depleted individuals will show greater subsequent self-control than non-depleted individuals (reversal of depletion effects).

\section{HEALTH MESSAGES AND DEPLETION EFFECTS}

In the domain of health communications, self-control is a relevant issue. Although past research on self-control has examined patient compliance (e.g., taking regular medication) and performing behaviors consistent with a healthy lifestyle (e.g., eating healthy, exercising regularly) as selfcontrol problems, little research has studied the processing of health messages from a self-control point of view (Menon, Raghubir, and Agrawal 2007). We suggest that processing health messages that create perceptions of high risk of contracting a disease may require self-control. Prior research has suggested that processing a high-risk health message presents a trade-off between long-term benefits and shortterm interests. Although individuals at high risk for a disease can improve their long-term well-being by taking preventative actions, recognizing risk leads to emotional deterioration in the short run (Agrawal and Duhachek 2008; Agrawal and Menon 2008; Agrawal, Menon, and Aaker 2007; Keller, Lipkus, and Rimer 2003; Menon et al. 2007). In addition, health decisions might be laden with emotionally difficult trade-offs between making a decision that will help reduce emotional anguish and one that will benefit longterm health (Kahn and Luce 2003). These extant findings in the health literature are consistent with the view that processing high-risk messages may involve self-control and can deplete substantial self-control resources. Following this assertion, our studies employ health messages as a selfcontrol context in two ways. First, exposure to messages that convey either high- or low-risk perceptions was used in the initial task to manipulate the level of depletion. Effectively, messages that create high-risk perceptions should be more depleting than those that create low-risk perceptions. Second, processing health messages that present aversive but beneficial risk information about a high-risk condition requires self-control resources and could serve as a measure of self-control. Hence, we measure individuals' subsequent self-control by having participants perform health behaviors requiring self-control (e.g., persistence at reading threatening but beneficial medical information, persistence at flossing teeth). 


\section{OVERVIEW OF EXPERIMENTS}

The proposed hypotheses were tested in two experiments. Both experiments adopted a two-task procedure widely used in the resource depletion literature (Baumeister et al. 1998; Muraven et al. 1998). The first task manipulated the level of initial resource depletion: it consumed either substantial or modest amounts of self-control resources. This task involved processing a message that conveyed either high or low risk of contracting hepatitis $\mathrm{C}$ (Menon, Block, and Ramanathan 2002). The second task tested subsequent selfcontrol. This task involved reading threatening but beneficial information about a condition unrelated to hepatitis $C$ that was pretested to consume a substantial amount of self-control resources. In addition, between the first and the second task, we manipulated participants' level of construal.

Experiment 1 examined the effect of construal levels on depletion effects in a context in which the second task provided valuable higher-level benefits, and it also tested the processes by which lower-level construals affect depletion effects. Experiment 2 extended the investigation to contexts in which the importance of the self-control task in achieving higher-level benefits varied across conditions. It also tested the process underlying self-control at higher construal levels.

\section{EXPERIMENT 1}

Experiment 1 had two objectives. First, we test the prediction that the depletion effect will occur only for individuals at lower levels of construal (hypothesis 1a). For higherlevel construals, we examine the hypothesis that individuals will exert substantial self-control, regardless of initial resource depletion, when the subsequent self-control task is important to their higher-level goals (hypothesis 2a). For this purpose, we first manipulated initial resource depletion by exposing participants to messages about hepatitis $C$ that induced either a high- or a low-risk perception of contracting this disease, followed by a mind-set manipulation that primed individuals to adopt either higher or lower levels of construal. Subsequent tasks involved self-control behaviors related to dental health (e.g., persistence at processing beneficial but threatening and boring oral health information, time spent flossing teeth) — a domain that was pretested to be important to our participants' health goals. We predict that in the lower-level construal condition, participants exposed to the high-risk message for hepatitis $\mathrm{C}$ would perform poorly in the subsequent dental health task, relative to those exposed to the low-risk message. In contrast, in the higherlevel construal condition, participants would perform equally well in the subsequent dental health task, regardless of whether they saw a high- or low-risk hepatitis $\mathrm{C}$ message.

Second, we test the underlying process by which lowerlevel construals affect self-control in resource depletion. For this purpose, we measured participants' feelings of tiredness after completing the initial task and examined its effect on subsequent self-control under different levels of construal. If lower-level construal influences subsequent self-control because of an enhanced focus on resources, tiredness should mediate the effect of initial depletion on subsequent selfcontrol at lower but not higher levels of construal (hypothesis 1b).

\section{Method}

Sixty undergraduate students from a midwestern university were each paid $\$ 10$ to participate in an experiment that employed a 2 (initial depletion: depleting [high-risk health message] vs. non-depleting [low-risk health message]) $\times 2$ (construal level: lower [near future] vs. higher [distant future]) between-subjects design. After reading a message about hepatitis $C$ that manipulated the initial level of depletion, participants received the construal level manipulation. Following the construal level manipulation, they completed the dependent measures of time spent reading an article about dental health and time spent flossing their teeth. Each of these steps is described below, along with the relevant pretests.

Health Messages Manipulate Initial Depletion. First, all participants read a three-page message about hepatitis $C$. The first page was an advertisement that highlighted the severe consequences of contracting hepatitis $\mathrm{C}$ and listed eight behaviors that could lead to contracting hepatitis $\mathrm{C}$. The second and third pages presented information describing the hepatitis $\mathrm{C}$ virus and its contraction, prevention, and treatment (Menon et al. 2002). The manipulation involved presenting different lists of risky behaviors associated with hepatitis $\mathrm{C}$. The depleting-high-risk message featured frequently performed behaviors that could lead to hepatitis $\mathrm{C}$ (e.g., not banding a cut, kissing). The non-depleting lowrisk message presented infrequently performed behaviors (e.g., getting a blood transfusion; Menon et al. 2002).

A pretest conducted among 38 participants from the same respondent pool confirmed that presenting the two types of messages about hepatitis $\mathrm{C}$ successfully varied the level of initial resource depletion. This pretest adopted the typical procedure used in the depletion literature to test whether a behavior depletes self-control resources (e.g., Baumeister et al. 1998). Participants first read one of the two health risk messages described above. Afterward, participants were asked to solve a set of anagrams. These anagrams were actually unsolvable, but participants did not suspect them to be so. In the depletion literature, this unsolvable anagram task is frequently used as a measure of subsequent selfcontrol (e.g., Muraven et al. 1998, study 2). Participants were told that they could take as much time and as many trials as they wanted and that they could stop at any time. In performing this task, participants need to keep trying to solve anagrams despite being fatigued and discouraged by several failed attempts. Here, self-control is characterized by overriding the impulsive desire to quit trying when faced with frustration. Consistent with past research, the number of minutes spent on these anagrams served as the self-control measure (e.g., Muraven et al. 1998). The results showed that participants who read the depleting-high-risk message spent significantly less time $(M=5.63$ minutes $)$ solving ana- 
grams than those who read the non-depleting low-risk message $(M=7.53$ minutes; $F(1,36)=4.54, p<.05)$. Thus, consistent with our assertion, processing a high-risk (vs. low-risk) message decreased subsequent self-control.

Construal Level Manipulation. In the main experiment, after completing the hepatitis $\mathrm{C}$ task, participants worked on a mind-set task that manipulated the construal levels. A mind-set manipulation requires participants to repeatedly construe things at higher or lower levels. Such repeated construals prime participants to think at higher versus lower levels of construal on subsequent tasks (Fujita et al. 2006). In this task, all participants were required to describe six activities in writing (e.g., watching TV). In the lower-level construal condition, they imagined engaging in these activities tomorrow (near future). In the higher-level construal condition, they imagined these activities as occurring next year (distant future; Liberman and Trope 1998).

To ascertain the efficacy of the temporal distance mindset manipulation, we conducted a pretest following procedures documented in past research (e.g., Fujita et al. 2006; Jain, Agrawal, and Maheswaran 2006; Jain et al. 2007; Liberman and Trope 1998; Trope and Liberman 2000). Fortytwo participants from the same respondent pool completed the above temporal distance manipulation followed by the Behavioral Identification Form (BIF; Vallacher and Wegner 1989). The BIF is a 25 -item dichotomous questionnaire that was initially designed to assess individual differences in the level at which people tend to identify. Individuals are asked to choose one of two descriptions of the same behavior (e.g., making a list), one that comprises higher-level construals related to goals or why considerations (e.g., getting things organized) and the other that comprises lower-level construals related to means or how considerations (e.g., writing something down). Past research has shown that the situational manipulation of individuals' mind-set, such as having individuals think about things happening in the distant future (e.g., next year) versus the near future (e.g., tomorrow), also systematically influences responses to the BIF questions (e.g., Fujita et al. 2006; Liberman and Trope 1998, study 1). In accord with this past research, we used the BIF to assess the efficacy of the construal level manipulation. A construal level score was formed for each participant by averaging the number of higher-level alternatives chosen such that higher scores indicate higher construal levels. Participants in the distant future condition had a significantly higher BIF score $(M=.61)$ than did those in the near future condition $(M=.49 ; F(1,40)=4.83, p<.04)$, confirming that temporal distance manipulation systematically varied individuals' construal level.

In an additional pretest to examine whether the temporal distance manipulation leads to a differential focus on means versus goals, 46 participants from the same respondent pool performed the temporal distance manipulation followed by an open-ended writing task in which they described their academic life (Liberman and Trope 1998). Two judges $(r=.91)$ categorized the sentences as goals related (why), means related (how), or other. As expected, participants in the distant future condition wrote more goal-related $(60 \%)$ rather than means-related descriptions $(23 \% ; p<.04)$. Conversely, participants in the near future condition wrote more means-related $(62 \%)$ rather than goal-related descriptions $(24 \% ; p<.04)$. These results support our assertion that temporal distance systematically influences participants' focus on goals versus means.

Measuring Subsequent Self-Control: Time Spent Reading about Dental Health. Following the construal level manipulation, participants read an article about dental health purportedly distributed by the university health center. This 2,123-word article described in medical terms the symptoms, prevention, and treatment of various dental diseases. Although this information was beneficial for dental health, it was threatening and boring, constituting a self-control problem. Persistence measured in minutes spent reading this article served as the dependent measure of self-control.

A pretest based on the depletion literature confirmed that reading the dental health article required substantial selfcontrol resources. According to resource depletion theory, if reading the dental health article involved self-control resources, then performing a prior unrelated task that is depleting (vs. non-depleting) in resources would reduce persistence in reading about dental health (e.g., Gailliot, Schmeichel, and Baumeister 2006; Vohs, Baumeister, and Ciarocco 2005). Based on this rationale, 42 participants in the pretest completed a cross-off-letters task that has been used in previous research to manipulate initial resource depletion (e.g., Baumeister et al. 1998, experiment 4), followed by the task of reading the target dental health article. In the non-depleting condition, the initial task involved crossing off all instances of the letter $e$ in a text. The depleting version of the task involved crossing off instances of $e$ in the same text but only when it met two criteria (i.e., $e$ was not adjacent to another vowel, and it was not one letter away from another vowel). Past research has suggested that participants tend to scan for each $e$ but need to override their impulse to cross off the letter when the specified criteria are not met. Compared with the non-depleting version, the depleting version has been shown to require substantially more self-control resources (Baumeister et al. 1998). The results of the pretest showed that participants who performed the depleting version of the cross-off-letters task spent less time reading the dental health article $(M=4.77)$ than those who performed the non-depleting version $(M=6.50 ; F(1,40)=4.98, p<$ $.04)$, confirming that reading the dental health article required self-control.

Another pretest was conducted to ensure that dental health was important to participants' health goals. In this pretest, 64 participants responded to two questions. Responses to "How important is it for you to be well informed about dental health issues?" on a scale anchored at 1 (not important at all) and 9 (very important) showed that participants in our study regarded dental health information as an important health topic $(M=7.41)$. Participants were also asked about the number of times they visit a dentist every year. On average, participants reported visiting the dentist twice every 
TABLE 1

RESULTS FOR EXPERIMENT 1: CONSTRUAL LEVELS AND DEPLETION INFLUENCE SUBSEQUENT SELF-CONTROL

\begin{tabular}{|c|c|c|c|c|}
\hline \multirow[b]{2}{*}{ Dependent measures } & \multicolumn{2}{|c|}{ Lower-level construal (near future) } & \multicolumn{2}{|c|}{ Higher-level construal (distant future) } \\
\hline & $\begin{array}{c}\text { Low-risk } \\
\text { health message } \\
\text { (non-depleting) }\end{array}$ & $\begin{array}{c}\text { High-risk } \\
\text { health message } \\
\text { (depleting) }\end{array}$ & $\begin{array}{c}\text { Low-risk } \\
\text { health message } \\
\text { (non-depleting) }\end{array}$ & $\begin{array}{c}\text { High-risk } \\
\text { health message } \\
\text { (depleting) }\end{array}$ \\
\hline \multicolumn{5}{|l|}{ Self-risk probability estimate for contracting hepatitis $C$} \\
\hline Feeling of tiredness after completing the hepatitis $C$ task & 3.51 & $4.95^{\star}$ & 4.62 & $5.68^{*}$ \\
\hline Persistence on reading about dental health (minutes) & 5.18 & $2.79^{\star *}$ & 5.04 & 4.97 \\
\hline Persistence on flossing (minutes) & 2.74 & $2.11^{*}$ & 3.29 & 3.12 \\
\hline
\end{tabular}

year $(M=2.01)$. These two insights confirmed that maintaining dental health was an important health goal for the participants in this study.

Measuring Subsequent Self-Control: Time Spent Flossing. In the main experiment, after participants read the dental health article, we collected a behavioral measure of self-control-persistence on flossing. Participants were taken one at a time to a separate room where floss picks, spring water, napkins, and a trash bin were provided. The experimenter asked participants to try the sample floss picks and left the room. Unknown to participants, the experimenter observed them through a narrow glass window and recorded the time spent on flossing.

Flossing was chosen to test self-control related to dental health because a pretest had shown that flossing required self-control. In this pretest, 27 participants answered two questions ( $\alpha=.85$ ) about the perceived benefits of flossing: "How good is flossing for your teeth?" and "How beneficial is flossing to your teeth?" They also answered three questions $(\alpha=.89)$ about the pleasantness of flossing: "How pleasant do you think flossing is?" "Is flossing a fun thing to do?" and "Do you like flossing?" Responses were collected on 9-point scales anchored at "not at all" and "a lot." The perceived benefit of flossing rated significantly higher $(M=7.72)$ than its pleasantness $(M=3.62 ; F(1,26)=$ $118.74, p<.001)$, suggesting that flossing featured a conflict between benefits and pleasantness and hence required selfcontrol.

Manipulation Check Measures. To help us ascertain whether the high- (vs. low-) risk message led to higher risk perceptions, participants assessed the probability that they might contract hepatitis $\mathrm{C}$ on a 101-point scale $(0=$ definitely would not have it, and $100=$ definitely would have it) immediately after exposure to the hepatitis $\mathrm{C}$ message. At the end of the study, participants completed an additional questionnaire. They were asked to think about the task of reading the hepatitis $\mathrm{C}$ message and indicate how tired they felt while reading it on a scale anchored at 1 (not tired at all) and 9 (very tired). As a manipulation check for the temporal distance manipulation, participants responded to the question, "When do you perceive those activities (e.g., watching TV) will occur?" on a scale anchored at 1 (tomorrow) and 9 (1 year from now). As controls for individual differences, participants provided their dental health history by indicating whether they had had problems with dental health and how carefully they usually flossed, on a scale ranging from 1 (not careful at all) to 9 (very careful). Then participants were debriefed, paid, and thanked.

\section{Results and Discussion}

Manipulation Checks. First, we examined participants' risk perceptions of contracting hepatitis $\mathrm{C}$. The $2 \times 2$ ANOVA revealed only a significant main effect of initial depletion, confirming the efficacy of high- versus low-risk message manipulation. As expected, the frequent behavior message led to greater perceived probability of contracting hepatitis $\mathrm{C}(M=22.75)$ than did the infrequent behavior message $(M=8.90 ; F(1,56)=11.23, p=.001)$. Validating the temporal distance manipulation, participants in the distant (vs. near) condition expected the six activities (e.g., watching TV) to occur further in the future $(M=$ 5.26 vs. $M=3.86 ; F(1,59)=6.53, p<.02)$.

Subsequent Self-Control: Persistence in Reading about Dental Health. A $2 \times 2$ ANOVA on the minutes spent reading about dental health revealed a significant main effect of initial depletion $(F(1,56)=4.50, p<.04)$ and its significant interaction with construal level $(F(1,56)=$ $4.01, p=.05$; see table 1$)$. In support of hypothesis $1 \mathrm{a}$, planned contrasts indicated that when primed with lowerlevel construals, participants who read a high-risk health message spent significantly less time reading about dental health $(M=2.79)$ than did those who read a low-risk health message $(M=5.18 ; F(1,56)=8.27, p<.01)$. Consistent with hypothesis $2 \mathrm{a}$, we found that at higher construal levels, for an important domain such as dental heath, participants exerted substantial self-control. Time spent reading about 
FIGURE 1

MEDIATION ANALYSIS (EXPERIMENT 1): TIREDNESS MEDIATES DEPLETION EFFECTS ONLY UNDER LOWER-LEVEL CONSTRUALS

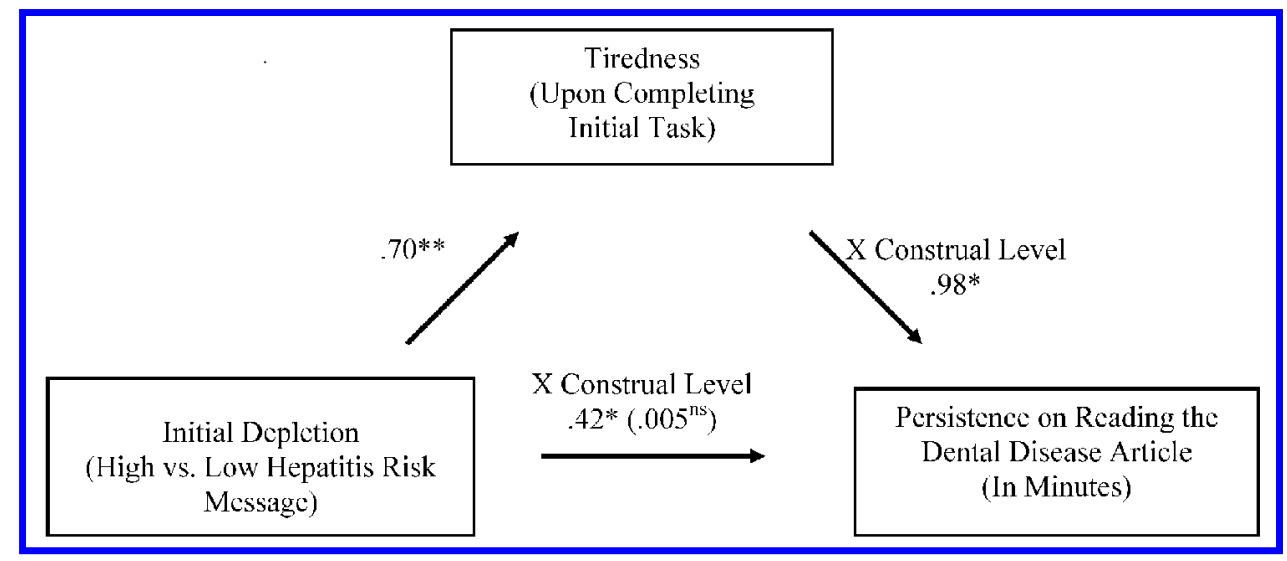

Note. - * significant at the .05 level; ${ }^{*}$ significant at the .01 level.

dental health did not vary as a function of high-risk $(M=4.97)$ or low-risk messages $(M=5.04 ; F<1)$. Further, among participants who read a high-risk message (i.e., depleted participants), those at lower construal levels spent significantly less time reading about dental health $(M=$ 2.79) than did those at higher levels $(M=4.97 ; F(1,56)=$ 8.67, $p<.02)$. Participants who read the low-risk message (i.e., non-depleted participants) did not vary in persistence as a function of construal level.

Tiredness as a Mediator. To test the process underlying the moderating effect of construal levels on the depletion effect (hypothesis 1b), we conducted a mediated moderation analysis following the procedure described in Muller, Judd, and Yzerbyt (2005) using feelings of tiredness as the mediator. First, we regressed persistence in reading about dental health on initial depletion (i.e., whether the hepatitis $\mathrm{C}$ message created high- or low-risk perceptions), construal level, and the interaction of these two variables. Consistent with the earlier ANOVA results, this regression revealed a significant main effect of initial depletion $(\beta=-.50, t(1,56)=-2.88, p<.01)$ and a significant interaction $(\beta=.42, t(56)=2.00, p=.05)$. Next, we regressed feelings of tiredness on initial depletion condition, construal level, and their interaction. Only the main effect of initial depletion was significant $(\beta=.70, t(56)=4.45$, $p<.001$ ), suggesting that regardless of construal level participants who completed a depleting rather than non-depleting task felt more tired. Finally, we regressed persistence in reading about dental health on initial depletion, construal level, the depletion $\times$ construal level interaction, tiredness, and the tiredness $\times$ construal level interaction. This regression resulted in a significant main effect of tiredness $(\beta=-.65, t(54)=-2.48, p<.02)$ and a significant tiredness $\times$ construal level interaction effect $(\beta=.98, t(54)=$ $2.30, p<.03)$. Notably, the interaction between initial de- pletion and construal level was no longer significant $(p>$ .90; Sobel test: $z=4.49, p<.001$; see fig. 1 ). These analyses show that the mediating role of tiredness on the depletion effect was qualified by the level of construal. Specifically, when Baron and Kenny (1986) mediations were run within each construal level condition, feelings of tiredness mediated the effect of initial depletion on subsequent self-control at lower-level construals but not at higher-level construals (hypothesis 1b).

Subsequent Self-Control: Time Spent Flossing. A $2 \times 2$ ANCOVA on the actual time (in minutes) spent flossing, with participants' dental history and flossing habits as covariates, yielded a significant interaction only between initial depletion and construal level $(F(1,51)=4.01, p=$ .05 ; see table 1$)$. Consistent with earlier results and hypothesis 1a, follow-up contrasts indicated that at lower construal levels, depleted participants (i.e., those who read a high-risk message) spent less time flossing $(M=2.11)$ than did non-depleted participants (i.e., those who read a lowrisk message; $M=2.74 ; F(1,51)=6.68, p<.05)$. At higher levels of construal, participants did not differ in flossing time $(F<1)$. These outcomes were such that depleted participants at lower levels of construal $(M=2.11)$ spent less time flossing than did those at higher levels $(M=$ $3.12 ; F(1,51)=3.49, p=.06)$.

Discussion. Findings from experiment 1 support our theorizing on how construal levels affect self-control in resource depletion. Depletion effects occurred only at lower levels of construal, and feelings of tiredness mediated the effect of initial depletion on subsequent self-control. The mediation analyses provided evidence that resource accessibility influences self-control only at lower levels of construal. At higher levels of construal, participants spent substantial time flossing and reading about dental health, 
regardless of the nature of the initial task. This result supports our theorizing that under higher levels of construal when the self-control activity is linked with important goals, participants exert self-control and are not affected by their current access to resources.

Experiment 1 demonstrated how construal levels influence depletion effects in a context in which the subsequent self-control task was perceived as important to participants' health goals. To better understand the processes underlying depletion effects, in the next experiment we examine the effect of construal levels on self-control in resource depletion while varying the importance of the subsequent selfcontrol task. Specifically, we modified the experiment 1 procedures such that for the second task participants read an article about an unfamiliar disease. The perceived importance of acquiring information from this article was varied. We expected individuals at higher construal levels to spend more time reading this article when it was important rather than unimportant for the pursuit of health goals, independent of initial resource depletion (hypothesis 2a). In a control condition in which an explicit cue about importance was absent, we expected the effort consumed by the initial task to serve as an indicator of effort goals for the second task, leading to a reversal of the depletion effect predicted in hypothesis 2c. At lower construal levels, subsequent selfcontrol in acquiring health information would be affected by the resource depletion in the initial task but would not vary by importance of the task. In addition, mediation analyses tested the processes underlying self-control at higher construal levels.

\section{EXPERIMENT 2}

Experiment 2 employed a procedure similar to experiment 1 with the following changes. First, we added a third independent variable that varied the importance of processing the disease information in the second task. Instead of discussing dental health, the health article presented in the second task described an unfamiliar disease. The importance of reading this information was varied across conditions to be high or low or absent (control).

Second, we measured participants' recall of information in the unknown disease article, in addition to persistence on reading this article. One might argue that spending more time on reading might not imply better self-control if participants were not carefully processing the message. Recall could capture careful processing of the message.

Third, we collected measures that would provide insights into the processes underlying depletion effects under both higher and lower levels of construal. To examine the mechanisms underlying self-control at higher construal levels, we measured participants' perceptions of higher-level goals served by reading about the unfamiliar disease. To test the proposition that at lower construal levels focusing on resource limitation underlies the effect of initial depletion on subsequent self-control, we collected a more nuanced measure of focus on resources. Experiment 1 measured participants' feelings of tiredness upon completing the initial task and found that this measure mediated the link between initial resource depletion and subsequent self-control at lower construal levels. In experiment 2, we measured participants' focus on tiredness, while exerting self-control on the subsequent health task. Finally, we used a different construal level manipulation based on Freitas, Gollwitzer, and Trope (2004).

\section{Method}

One hundred and seventy undergraduate students from a midwestern university were each paid $\$ 10$ to participate in an experiment that employed a 2 (initial depletion: depleting [high-risk health message] vs. non-depleting [low-risk health message]) $\times 2$ (construal level: lower [how mind-set] vs. higher [why mind-set]) $\times 3$ (importance of exerting selfcontrol on the subsequent task: high vs. low vs. control) between-subjects design. Participants first completed the same hepatitis $\mathrm{C}$ task used in experiment 1 and estimated their risk of contracting hepatitis $\mathrm{C}$ on a $0-100$ probability scale.

Mind-Set Manipulation for Construal Levels. After reading about hepatitis $\mathrm{C}$, participants completed a mindset manipulation based on Freitas et al. (2004), which has been used to vary construal levels successfully in previous literature (e.g., Agrawal, Trope, and Liberman 2008; Fujita et al. 2006). Participants in the how (why) mind-set condition completed a study titled "How (Why) I do the things I do." In the how mind-set condition, participants reflected on how they would go about maintaining good personal relationships. The task was illustrated with the example of "attaining life happiness" that provided detailed descriptions of how bigger goals are fulfilled by smaller tasks. For example, for the goal of being happy in life, how can one move toward being happy-by getting a good job? How can one get a good job-by earning a degree? How does one earn a degree-by completing course requirements? Participants were told to think and write about "maintaining good personal relationships" in a similar way. Likewise, in the why mind-set condition, participants reflected on why they would maintain good personal relationships (Freitas et al. 2004). A separate pretest $(n=23)$ examined the efficacy of the why versus how mind-set manipulation in the same participant population. In the pretest, after performing the how or why mind-set task, participants completed the BIF (Vallacher and Wegner 1989) described earlier. Confirming the efficacy of the manipulation, the why mind-set $(M=$ .69) resulted in a significantly higher level of construal than the how mind-set $(M=.52 ; F(1,22)=4.28, p<.05)$.

Task Importance Manipulation and Subsequent SelfControl. After the mind-set manipulation, participants estimated their probability of contracting hepatitis $\mathrm{C}$ again. It is possible that why (vs. how) mind-sets might make a health risk appear distant and consequently reduce participants' risk perceptions (Chandran and Menon 2004). To test whether changes in construal levels alter participants' risk percep- 
tions, we measured their risk estimation both before and after the mind-set manipulation. Participants were then presented with a 1,616-word article that described the symptoms, prevention, and treatment of a disease unfamiliar to the target population. The disease, which was given a fictitious name, had symptoms that could occur to anyone. The article was designed to be threatening and extremely boring. The importance of reading this article to participants' health goals was manipulated by the relevance of the prevention and treatment information to the participants' age group. The information was presented as being highly beneficial either to 15-25-year-olds (high-importance condition) or to those older than 40 years (low-importance condition). In the control condition, we omitted any reference to the importance of information based on age groups.

Time spent on reading this article was recorded as a measure of subsequent self-control. Next, participants were asked to indicate the extent to which they thought about feeling tired while reading about the unfamiliar disease. To examine whether participants saw the information as related to their higher-level goals, we assessed how important, beneficial, and useful they thought acquiring information from the article was. These questions were anchored on scales ranging from 1 (not at all) to 9 (a lot). As an additional measure of self-control, participants then recalled the prevention methods described in the article. To control for individual differences in health concerns, participants also reported the frequency of their visits to a physician. Finally, participants were debriefed, paid, and thanked.

\section{Results and Discussion}

Manipulation Checks. First, we examined participants' perceived risk of contracting hepatitis $\mathrm{C}$ before the construal level manipulation. A one-way ANOVA showed that frequent $(M=29.12)$ versus infrequent behaviors $(M=4.18)$ led to greater risk estimates $(F(1,167)=20.68, p<.001)$. A twoway ANOVA with initial depletion and construal level as independent variables showed that risk perceptions collected after the construal level manipulation replicated the earlier findings that frequent rather than infrequent behaviors led to higher risk perceptions $(M=27.97$ vs. $M=13.26$; $F(1,164)=24.29, p<.001)$. Thus, high- versus low-risk manipulations were successful, and construal levels did not alter risk perceptions.

Subsequent Self-Control: Persistence in Reading about an Unfamiliar Disease. A $2 \times 2 \times 3$ ANCOVA on time spent reading about the previously unfamiliar disease, with frequency of physician visits as a covariate, produced the following significant effects: a main effect of construal level $(F(1,146)=4.15, p<.05)$, an interaction between construal level and task importance $(F(2,146)=$ $6.99, p=.001)$, an interaction between construal level and depletion $(F(1,146)=23.57, p<.001)$, and the key threeway interaction $(F(2,146)=3.50, p<.04$; see table 2 and fig. 2 for means).

Follow-up analyses revealed that at lower construal levels, depleted participants (i.e., those who read a high-risk health message) spent significantly less time on reading the unfamiliar disease article $(M=3.02)$ than did non-depleted participants (i.e., those who read a low-risk health message), regardless of the task importance cue $(M=4.35$; $F(1,146)=23.75, p<.001)$.

In contrast, at higher construal levels, persistence in reading the article about the unfamiliar disease varied across conditions. When acquiring information about the unfamiliar disease was known to be important or unimportant to the participants, depleted and non-depleted participants did not differ in their persistence in reading the disease article $(F$ 's $<1)$. That is, replicating results from experiment 1 , when the task importance was clear, initial depletion had no effect on participants' subsequent self-control. Validating the importance manipulation, participants showed greater selfcontrol in the high-importance conditions $(M=4.90)$ than in the low-importance conditions $(M=3.36 ; F(1,146)=$ $19.08, p<.001)$, regardless of initial depletion. These results show that for participants at higher construal levels, persistence in reading the disease article was not affected by the resource depletion that occurred in the initial task. When the importance of the second task was clear, their self-control depended on whether exerting self-control was important to achieving their health goals (hypothesis 2a).

When the importance of reading about the unfamiliar disease was unclear (i.e., in the control condition), results supported hypothesis 2c. Depleted participants spent significantly more time reading the article about the unfamiliar disease $(M=5.10)$ than non-depleted participants $(M=$ 3.06; $F(1,146)=15.09, p<.001)$. When the importance of the second task to goals was unclear, participants were likely to rely on the most salient effort cue (e.g., previous task) to determine the self-control they should devote to the second task. Consequently, depleted rather than non-depleted participants exerted more self-control, resulting in the reversal of the depletion effect.

Process under Lower Levels of Construal: Focus on Tiredness. To further test the process in hypothesis $1 \mathrm{~b}$, we examined whether focusing on tiredness mediated the effect of resource depletion on subsequent self-control under lower-level construals, following Baron and Kenny's (1986) procedure. In the lower-level construals condition, we first regressed persistence in reading the unknown disease article on initial resource depletion (i.e., whether the hepatitis $\mathrm{C}$ message created high- or low-risk perception). The results indicated a significant relationship between the two variables $(\beta=-.47, t(1,84)=-4.88, p<.001)$. Then a regression with focus on tiredness as the dependent variable and initial depletion as the independent variable showed that greater initial depletion led to a greater focus on tiredness $(\beta=$ $.43, t(1,84)=4.41, p<.001)$. Next, we regressed persistence on focus on tiredness and found that a greater focus on tiredness led to less persistence $(\beta=-.66, t(1,84)=$ $8.14, p<.001)$. Finally, we regressed persistence on both initial depletion and focus on tiredness. Both initial depletion $(\beta=-.22, t(1,83)=-2.56, p<.05)$ and focus on tired- 


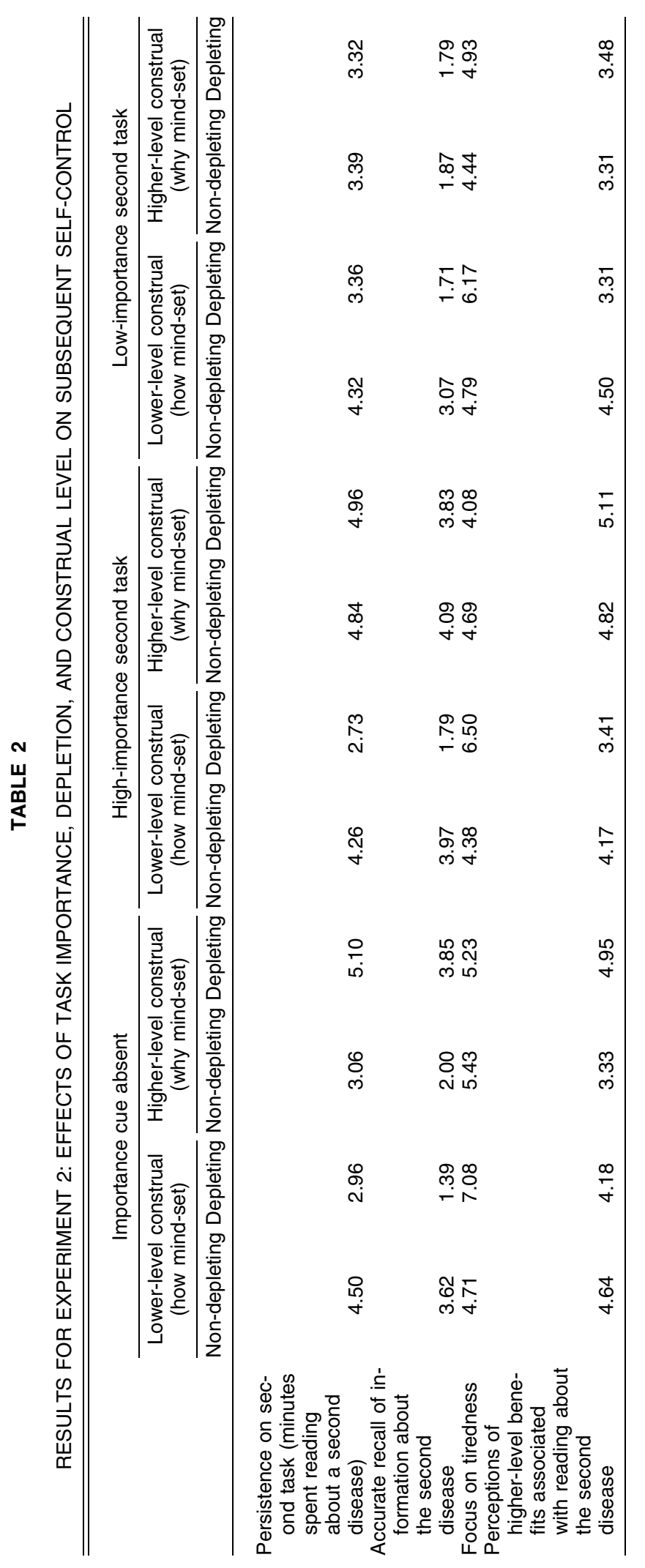


FIGURE 2

EFFECTS OF TASK IMPORTANCE, DEPLETION, AND CONSTRUAL LEVEL ON PERSISTENCE OF READING DISEASE ARTICLE (EXPERIMENT 2)

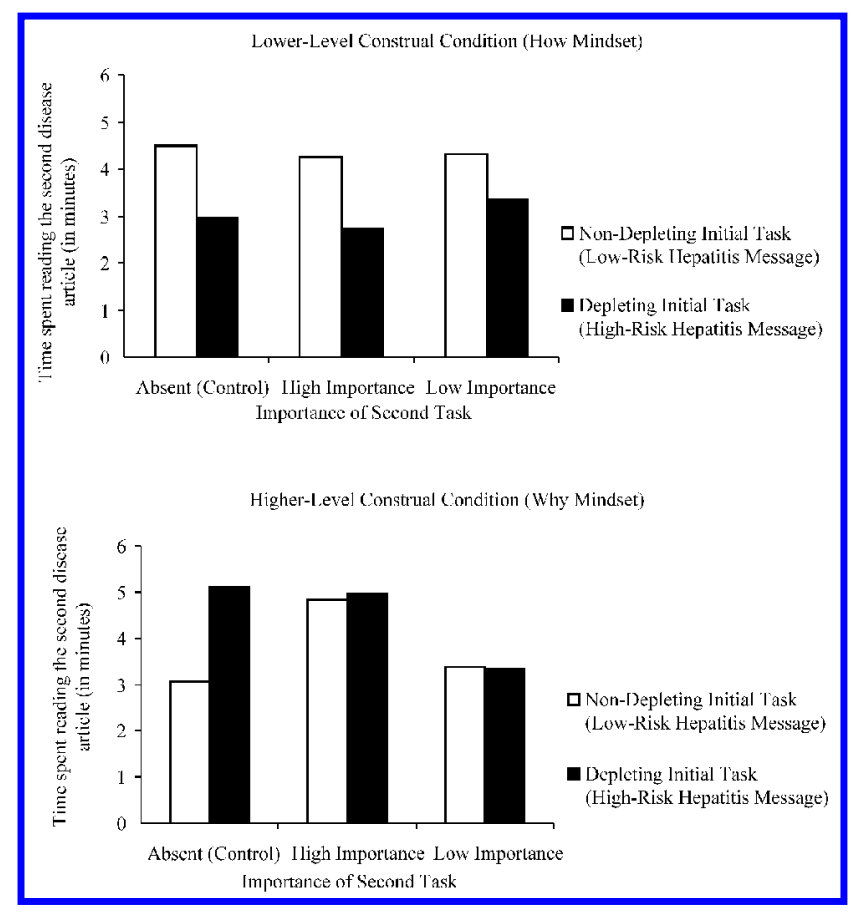

ness $(\beta=-.57, t(1,83)=-6.47, p<.001)$ were significantly related to persistence. However, the coefficient of initial depletion significantly decreased, relative to when only initial depletion was used to predict persistence (Sobel test: $z=3.88, p<.001$ ), suggesting that at lower construal levels the effect of initial depletion from performing the initial task on subsequent self-control is mediated by a focus on tiredness. At higher construal levels, we ran similar analyses involving focus on tiredness as the mediator. As expected, tiredness did not influence subsequent self-control.

Process under Higher Levels of Construal: Perceived Higher-Level Benefits. To test the process in hypothesis $2 \mathrm{~b}$, a perceived higher-level benefits index was obtained by averaging responses to the perceived importance, benefits, and usefulness of the information about the unfamiliar disease $(\alpha=.90)$. Two separate mediation analyses examined how perceived task importance influenced the experimental effects on self-control at higher construal levels.

The first set of mediation analyses examined the underlying process when the importance of self-control in the subsequent task was made explicit. Our results show that for participants primed with higher-level construals in the high- and low-task-importance conditions, subsequent selfcontrol at higher construal levels was affected by the manipulation of task importance and not by resource depletion in the initial task. Thus, for participants at higher construal levels we examined whether perceived benefits mediated the effect of manipulated task importance on subsequent selfcontrol. A series of regressions supported the above mediation (Baron and Kenny 1986). Regressions confirmed that when task importance was manipulated to be high versus low, participants were more persistent in the subsequent task $(\beta=.52, t(1,53)=4.46, p<.001)$, the manipulations of task importance influenced participants' perceived benefits of reading the unfamiliar disease article $(\beta=.49, t(1,53)=$ $4.08, p<.001)$, and perceived benefits predicted subsequent self-control $(\beta=.75, t(1,53)=8.14, p<.001)$. Finally, when the task importance manipulation and perceived benefits were both included as independent variables to predict subsequent self-control, perceived benefits remained a significant predictor $(\beta=.66, t(1,52)=6.39, p<.001)$, whereas the task importance cue became nonsignificant $(\beta=.18$, $t(1,52)=1.74, p=.08$; Sobel test: $z=3.65, p<.001)$. These mediation analyses support our theorizing that when exerting self-control is clearly important to participants' goals, participants rely on this importance information, and initial resource depletion plays no role in determining subsequent self-control. Consistent with our theorizing, similar mediation analyses performed in the lower-level construal conditions did not achieve significance.

A second set of mediation analyses was conducted within the control condition to examine the underlying process when the importance of exerting self-control on the subsequent task was unclear. In this condition, we expected participants to rely on the effort spent on the previous task to estimate the effort they should put into the subsequent task. Consistent with this expectation, as reported earlier, depleted participants exerted more self-control than nondepleted ones. That is, when persistence in reading was regressed on initial depletion, as reported earlier, greater depletion led to greater persistence $(\beta=.57, t(1,25)=$ $3.44, p<.01)$. Hence, we followed up to examine whether depletion on the first task influenced perceived higher-level benefits from the second task, which in turn determined subsequent self-control. When the perceived benefits measure was regressed on initial depletion, greater initial depletion resulted in higher perceptions of benefits $(\beta=.41$, $t(1,25)=2.24, p<.04)$. We also found that perceived benefits predicted subsequent persistence $(\beta=.75, t(1,25)=$ $5.72, p<.001)$. Finally, we regressed persistence on both initial depletion and perceived higher-level benefits. Although both initial depletion $(\beta=.31, t(1,24)=2.35$, $p<.03)$ and perceived benefits $(\beta=.63, t(1,24)=4.71$, $p<.001)$ remained as significant predictors, the coefficient of initial depletion was significantly smaller than when only initial depletion was used to predict persistence (Sobel test: $z=2.05, p<.01)$. These results suggest that at higher construal levels, perceived benefits from exerting subsequent self-control mediated the effect of initial depletion on subsequent self-control in the control condition. For ease of exposition, the mediation analyses for this experiment were conducted following Baron and Kenny's (1986) models. Similar analyses using mediated moderation models (Muller et al. 2005) provided convergent results. 
Effectiveness of Persistence: Accurate Recall of Information. To examine whether spending a greater amount of time on reading the disease article reflected greater selfcontrol in processing beneficial but threatening and unpleasant information, we analyzed the effect of experimental treatments on participants' accurate recall of disease prevention information. The results replicated the findings on the persistence measure. The key interactions and simple effects on the recall measure were significant and consistent with results on the persistence measure reported earlier. Due to space constraints, these data are not reported in detail here. The means for this measure are reported in table 2 .

Discussion. Experiment 2 replicated the depletion effect at lower construal levels, demonstrating again that this effect was mediated by a focus on current access to resources. At higher construal levels, the depletion effect did not occur, and self-control was determined by the importance of the second task to individuals' goals. Replicating the results of experiment 1 , depleted and non-depleted participants both had equivalent and high self-control on the second task when this task was framed as high in importance. Both depleted and non-depleted participants had equivalent but poor subsequent self-control when the second task was framed as low in importance. These effects were mediated by participants' perceptions of higher-level benefits to be attained from exerting self-control on the second task. When the importance of the second task to participants' health goals was unclear (control condition), a reversal of the depletion effect emerged. Hence, experiment 2 documented the robustness of our predicted effects and provided direct evidence for the processes underlying these effects.

\section{GENERAL DISCUSSION}

The current research examines the effect of construal level on self-control from the resource depletion perspective. We proposed that lower-level construals emphasize means and resources and therefore undermine self-control when the initial task is resource depleting. In contrast, higher-level construals emphasize goals and dilute the focus on resources. Hence, they are unlikely to impair self-control as a consequence of depletion in resources. Results from two experiments support this theorizing. The detrimental effect of resource depletion on self-control occurred only for individuals at lower levels of construal. Moreover, the processes underlying these two effects were examined. A focus on resource limitations, specifically feelings of tiredness, mediated the effect of initial resource depletion on subsequent self-control for individuals at lower construal levels. Despite experiencing similar levels of tiredness as those at lower construal levels, the self-control exhibited by participants at higher levels of construal was not adversely affected by their feelings of tiredness. At higher construal levels, individuals' perceptions of the benefits to goals attained by performing the second task mediated the treatment effects on subsequent self-control. These findings add to literatures on self-control, construal levels, and health risk perceptions.
Construal Levels and Self-Control. Previous research on the relationship between construal levels and self-control has shown that higher-level construals highlight global goals to facilitate self-control, whereas lower construal levels highlight local goals leading to impaired self-control (Fujita et al. 2006). This line of research has examined situations in which participants primed with high or low construal levels faced a self-control task - these are contexts in which only one self-control task is performed. However, past research has shown that the outcomes and processes that determine self-control in consecutive tasks-these are contexts in which participants are faced with one self-control task after having already performed a previous self-control task (e.g., the depletion effects paradigm; Novemsky and Dhar 2005; Vohs and Faber 2007) - might be different from those that determine self-control when participants perform only one self-control task. The current research extends prior research by examining the effects of construal levels on selfcontrol in the depletion paradigm in which individuals must perform two consecutive self-control activities. In doing so, we identify a different route through which construal levels influence self-control.

We employ the goals-means distinction among construal levels to make predictions about self-control associated with the depletion effect. According to CLT, higher-level construals comprise desirability aspects and goals. Consistent with Fujita et al. (2006), our findings showed that higherlevel construals influence self-control, depending on whether the focal task is important to the pursuit of higher-level goals. We extend the Fujita et al. (2006) finding in two ways. First, we document that the finding holds for depleted participants, which is critical because previous research on resource depletion has argued that goals might not matter for depleted participants (Vohs and Schmeichel 2003, 219). Second, while past research has suggested that higher-level construals are driven by goals, we show that when goals are unclear, salient means-related information (e.g., tiredness from a previous task) might serve as a benchmark to infer goals for subsequent tasks (e.g., the control condition in experiment 2). In doing so, we show a reversal of previous research that argues that higher rather than lower levels of construal lead to greater self-control (Fujita et al. 2006). The present research documents the opposite effect in experiment 2: higher-level $(M=3.06)$ construals lead to lower selfcontrol relative to lower-level construals (compare the first white bar in the top and bottom panels in fig. 2; $M=$ $4.50 ; F(1,146)=9.87, p<.01)$.

CLT has proposed that lower-level construals are richer and more multidimensional than higher-level construals. They comprise local goals, feasibility concerns, means, resources, constraints, and so on (Trope and Liberman 2003). For lower construal levels, our predictions and results differ from Fujita et al. (2006). Fujita et al. (2006) propose a localgoals-driven mechanism of how lower levels of construal affect self-control, which predicts that lower-level construals undermine self-control. We propose a resource-focused mechanism through which lower-level construals influence 
self-control. That is, at lower construal levels, self-control is determined by individuals' focus on the means and resources currently accessible to them. Hence, depleted individuals tend to focus on the resource limitation (e.g., enhanced feelings of tiredness) and show reduced subsequent self-control. For non-depleted individuals, in contrast to Fujita et al.'s finding (2006), we find that lower levels of construal led to an assessment of plentiful resources and did not undermine self-control. This result that lower levels of construal may not necessarily lead to self-control failure differs from Fujita et al.'s (2006) prediction but is consistent with previous research on constructs related to lower-level construals. For example, implementation intentions, which focus on how one will deal with goal pursuit challenges, are construed at lower levels and have been shown to increase self-control (Gollwitzer 1999). Our research also differs from Fujita et al. (2006) in that they examine the effect of construal levels on self-control given the attributes of that self-control dilemma (e.g., higher- vs. lower-level benefits of watching a movie over studying). Our analysis illustrates how construal levels might systematically allow person-specific attributes that are unrelated to the self-control dilemma (i.e., extent of tiredness from a previous task) to influence self-control.

It is also noteworthy that although CLT posits that lowerlevel construals incorporate several aspects (e.g., local goals, means), it does not make specific predictions about differences in the outcomes resulting from means-driven versus local-goals-based mechanisms. The underlying assumption in previous research has been that different aspects of lowerlevel construals (e.g., means vs. local goals) lead to the same outcome. Our research suggests that these two aspects of lower-level construals may lead to different outcomes. Thus, not only does our documentation of lower-level construals affecting self-control through a resource-based mechanism present an advance over Fujita et al.'s (2006) findings on self-control, but it also enriches the current framework of CLT.

Our studies highlight several avenues for future research in this area. One important question pertains to when localgoals- versus means-related concerns would drive behavior under lower construal levels. Follow-up studies should investigate whether means-related concerns are more salient in consecutive-task settings than in single-task setups. The nature of the self-control trade-offs involved might also determine whether the process is driven by goals or means. Most of our studies as well as studies in the depletion literature use grim necessities as depleting initial tasks, whereas other work on self-control relies on guilty pleasures (Giner-Sorolla 1999). Future research should examine the possibility that grim necessities are governed by means, whereas guilty pleasures are driven by local goals.

Depletion Effects and Self-Control. Findings from the current research enrich the literature on resource depletion in two ways. First, by using construal levels as a moderator, we add to the research demonstrating that depletion effects could be overcome without replenishing resources (Muraven and Slessareva 2003; Wan and Sternthal 2008). In an experiment not reported in this article, we replicated the moderating effect of construal levels on depletion effects employing experimental tasks that are typically used in the depletion literature (a cross-off-letters task followed by an anagram task). Second, by implicating construal levels, we add to the understanding of the mechanism underlying depletion effects. Higher-level construals diluted the focus on resources and enhanced a focus on goals, which helped individuals in exerting self-control despite being initially depleted. In contrast, lower-level construals enhanced the focus on resources (e.g., tiredness), which led to depletion effects. Mediation analyses show that focusing on resource limitation (e.g., tiredness) leads to subsequent self-control failure. In sum, our research extends past research on resource depletion theory by showing that depletion effects are driven by not necessarily a lack of resources but a tendency to focus on seemingly depleted resources. Essentially, we show that depletion effects are not a problem of resource capacity but rather a problem of resource allocation. Interventions that diminish the focus on resources and enhance the focus on important goals could mitigate the depletion effect by facilitating virtuous allocation of resources. When consumers are feeling highly depleted, higher-level construals can facilitate self-control by motivating them to access the "reserve" of resources. Future research could examine interventions that might dilute a resource focus and encourage lower-level construals to adopt a goal focus (e.g., implementation mind-sets [Gollwitzer 1999], positive affect [Wan, Isen, and Sternthal 2008]).

Health Communications, Risk Perceptions, and SelfControl. The current findings are interesting from a health communication point of view. They emphasize that caution should be employed when persuading people to believe they are at high risk. Providing information about frequent (vs. infrequent) behaviors related to hepatitis $\mathrm{C}$ has been found effective in increasing self-risk estimates and behavioral intentions associated with hepatitis C (Menon et al. 2002; Menon, Kyung, and Agrawal 2009). However, our results suggest that while such information may be effective with respect to hepatitis $\mathrm{C}$, it might lead to subsequent unhealthy behaviors (e.g., undermining healthy behaviors such as flossing) in unrelated domains. In a follow-up study that examined the effect of exposure to health risk messages on food consumption, we manipulated initial depletion using the hepatitis $\mathrm{C}$ task and then varied mind-sets. We then measured the number of cookies participants consumed while completing an unrelated filler task. Results from this study revealed a significant interaction $(F(1,164)=4.51$, $p<.05)$ such that at lower construal levels, participants who read a high-risk message ate more cookies $(M=$ 1.88) than those who read a low-risk message $(M=$ $1.45 ; F(1,164)=5.82, p<.05)$. At higher levels of construal, the number of cookies consumed did not vary as a function of a high-risk $(M=1.31)$ or a low-risk message $(M=1.41 ; F<1)$. Further, participants who read a highrisk message consumed fewer cookies at higher-level 
rather than lower-level construals ( $M=1.31$ vs. 1.88; $F(1,164)=9.90, p<.01)$. This outcome suggests conditions in which exposure to messages that enhance risk perceptions has deleterious effects on subsequent self-control. Higher construal levels might mitigate such detrimental effects of processing high-risk messages. Our results suggest that when high risk is communicated in contexts in which consumers might face a series of unrelated self-control challenges, it would be beneficial to prime consumers with higher levels of construal by talking about long-term goals or to prime them with why mind-sets. Further research is needed to identify interventions that reduce the potentially harmful effects of well-intended health risk messages.

\section{REFERENCES}

Agrawal, Nidhi and Adam Duhachek (2008), "Emotional Compatibility and the Effectiveness of Anti-drinking Messages," working paper, Northwestern University, Evanston, IL 60201.

Agrawal, Nidhi and Geeta Menon (2008), "Harboring Hope and Accepting Anxiety: The Role of Uncertain Emotions in Judgments of Health Risk," working paper, Northwestern University, Evanston, IL 60201.

$\rightarrow$ Agrawal, Nidhi, Geeta Menon, and Jennifer L. Aaker (2007), "Getting Emotional about Health," Journal of Marketing Research, 44 (February), 100-113.

Agrawal, Nidhi, Yaacov Trope, and Nira Liberman (2008), "Value from Highlighting Time-Appropriate Outcomes," working paper, Northwestern University, Evanston, IL 60201.

$\rightarrow$ Baron, Reuben M. and David A. Kenny (1986), "The ModeratorMediator Variable Distinction in Social Psychological Research: Conceptual, Strategic, and Statistical Considerations," Journal of Personality and Social Psychology, 51 (December), 1173-82.

$\rightarrow$ Baumeister, Roy F., Ellen Bratslavsky, Mark Muraven, and Dianne M. Tice (1998), "Ego Depletion: Is the Active Self a Limited Resource?" Journal of Personality and Social Psychology, 74 (5), 1252-65.

$\rightarrow$ Chandran, Sucharita and Geeta Menon (2004), "When a Day Means More than a Year: Effects of Temporal Framing on Health Risk Judgments," Journal of Consumer Research, 31 (September), 375-89.

$\rightarrow$ Freitas, Antonio L., Peter Gollwitzer, and Yaacov Trope (2004), "The Influence of Abstract and Concrete Mindsets on Anticipating and Guiding Others' Self-Regulatory Efforts," Journal of Experimental Social Psychology, 40 (November), 739-52.

$\rightarrow$ Fujita, Kentaro, Yaacov Trope, Nira Liberman, and Maya LevinSagi (2006), "Construal Levels and Self-Control," Journal of Personality and Social Psychology, 90 (3), 351-67.

$\rightarrow$ Gailliot, Matthew T., Roy F. Baumeister, C. Nathan DeWall, Jon K. Maner, E. Ashby Plant, Dianne M. Tice, Lauren E. Brewer, and Brandon J. Schmeichel (2007), "Self-Control Relies on Glucose as a Limited Energy Source: Willpower Is More than a Metaphor," Journal of Personality and Social Psychology, 92 (2), 325-36.

$\rightarrow$ Gailliot, Matthew T., Brandon J. Schmeichel, and Roy F. Baumeister (2006), "Self-Regulatory Processes Defend against the Threat of Death: Effects of Self-Control Depletion and Trait Self-Control on Thoughts and Fears of Dying," Journal of Personality and Social Psychology, 91 (1), 49-62.

Giner-Sorolla, Roger (1999), "Affect in Attitude: Immediate and
Deliberative Perspectives," in Dual Process Theories in Social Psychology, ed. Shelly Chaiken and Yaacov Trope, New York: Guilford, 441-61.

Gollwitzer, Peter M. (1999), "Implementation Intentions: Strong Effects of Simple Plans," American Psychologist, 54 (July), 493-503.

Jain, Shailendra P., Nidhi Agrawal, and Durairaj Maheswaran (2006), "When More Is Less: Regulatory Focus and Responses to Maximal and Minimal Comparative Frames," Journal of Consumer Research, 33 (June), 91-98.

$\rightarrow$ Jain, Shailendra P., Charles Lindsey, Nidhi Agrawal, and Durairaj Maheswaran (2007), "For Better or for Worse? Valenced Comparative Frames and Regulatory Focus," Journal of Consumer Research, 34 (June), 57-65.

$\rightarrow$ Kahn, Barbara E. and Mary Frances Luce (2003), "Understanding High-Stakes Consumer Decisions: Mammography Adherence Following False Alarm Test Results," Marketing Science, 22 (3), 393-410.

Keller, Punam A., Isaac M. Lipkus, and Barbara K. Rimer (2003), "Affect, Framing, and Persuasion," Journal of Marketing Research, 40 (February), 54-65.

Kruger, Justin, Derrick Wirtz, Leaf Van Boven, and T. William Altermatt (2004), "The Effort Heuristic," Journal of Experimental Social Psychology, 40 (January), 91-98.

$\rightarrow$ Liberman, Nira and Yaacov Trope (1998), "The Role of Feasibility and Desirability Considerations in Near and Distant Future Decisions: A Test of Temporal Construal Theory," Journal of Personality and Social Psychology, 75 (July), 5-18.

$\rightarrow$ Menon, Geeta, Lauren Block, and Suresh Ramanathan (2002), "We're at as Much Risk as We're Led to Believe: Effects of Message Cues on Judgments of Health Risk," Journal of Consumer Research, 28 (March), 533-49.

$\rightarrow$ Menon, Geeta, Ellie Kyung, and Nidhi Agrawal (2009) "Biases in Social Comparison: Optimism or Pessimism?" Organizational Behavior and Human Decision Processes, 108 (January), 39-52.

Menon, Geeta, Priya Raghubir, and Nidhi Agrawal (2007), "Health Risk Perceptions and Consumer Psychology," in The Handbook of Consumer Psychology, ed. Curtis Haugtvedt, Paul Herr, and Frank Kardes, Philadelphia: Erlbaum, 981-1010.

$\rightarrow$ Muller, Dominique, Charles M. Judd, and Vincent Y. Yzerbyt (2005), "When Moderation Is Mediated and Mediation Is Moderated," Journal of Personality and Social Psychology, 89 (December), 852-63.

$\rightarrow$ Muraven, Mark and Roy F. Baumeister (2000), "Self-Regulation and Depletion of Limited Resources: Does Self-Control Resemble a Muscle?" Psychological Bulletin, 126 (March), 247-59.

$\rightarrow$ Muraven, Mark, Roy F. Baumeister, and Dianne M. Tice (1999), "Longitudinal Improvement of Self-Regulation through Practice: Building Self-Control Strength through Repeated Exercise," Journal of Social Psychology, 139 (4), 446-57.

$\rightarrow$ Muraven, Mark, R. Lorraine Collins, and Kristen Nienhaus (2002), "Self-Control and Alcohol Restraint: Test of the Self-Control Strength Model," Psychology of Addictive Behaviors, 16 (2), 113-20.

$\rightarrow$ Muraven, Mark and Elisaveta Slessareva (2003), "Mechanisms of Self-Control Failure: Motivation and Limited Resources," Personality and Social Psychology Bulletin, 29 (July), 894-906.

$\rightarrow$ Muraven, Mark, Dianne M. Tice, and Roy F. Baumeister (1998), "Self-Control as Limited Resource: Regulatory Depletion Patterns," Journal of Personality and Social Psychology, 74 (3), 774-89.

$\rightarrow$ Mussweiler, Thomas (2003), "Comparison Processes in Social 
Judgment: Mechanisms and Consequences," Psychological Review, 110 (3), 472-89.

$\rightarrow$ Novemsky, Nathan and Ravi Dhar (2005), "Goal Fulfillment and Goal Targets in Sequential Choice," Journal of Consumer Research, 32 (December), 396-404.

$\rightarrow$ Sagristano Michael D., Yaacov Trope, and Nira Liberman (2002), "Time-Dependent Gambling: Odds Now, Money Later," Journal of Experimental Psychology: General, 131 (3), 364-76.

$\rightarrow$ Trope, Yaacov and Nira Liberman (2000), "Temporal Construal and Time-Dependent Changes in Preference," Journal of Personality and Social Psychology, 79 (6), 876-89.

$\rightarrow-$ (2003), "Temporal Construal," Psychological Review, 110 (July), 403-21.

$\rightarrow$ Trope, Yaacov, Nira Liberman, and Cheryl Wakslak (2007), "Construal Levels and Psychological Distance: Effects on Representation, Prediction, Evaluation, and Behavior," Journal of Consumer Psychology, 17 (2), 83-95.

$\rightarrow$ Vallacher, Robin R. and Daniel M. Wegner (1987), "What Do People Think They're Doing? Action Identification and Human Behavior," Psychological Review, 94 (1), 3-15.

$\rightarrow$ (1989), "Levels of Personal Agency: Individual Variation in Action Identification," Journal of Personality and Social Psychology, 57 (October), 660-71.

$\rightarrow$ Vohs, Kathleen D., Roy F. Baumeister, and Natalie J. Ciarocco (2005), "Self-Regulation and Self-Presentation: Regulatory Resource Depletion Impairs Impression Management and Effortful Self-Presentation Depletes Regulatory Resources," Journal of Personality and Social Psychology, 88 (4), 632-57.

$\rightarrow$ Vohs, Kathleen D. and Ronald J. Faber (2007), "Spent Resources: Self-Regulation Resource Availability Affects Impulse Buying," Journal of Consumer Research, 33 (March), 537-47.

$\rightarrow$ Vohs, Kathleen D. and Brandon Schmeichel (2003), "Self-Regulation and the Extended Now: Controlling the Self Alters the Subjective Experience of Time," Journal of Personality and Social Psychology, 85 (August), 217-30.

Wan, Echo Wen, Alice M. Isen, and Brian Sternthal (2008), "The Influence of Positive Affect on Regulatory Depletion," working paper, the University of Hong Kong, Hong Kong.

$\rightarrow$ Wan, Echo Wen and Brian Sternthal (2008), "Regulating the Effects of Depletion through Monitoring," Personality and Social Psychology Bulletin, 34 (1), 47-60. 